\title{
Thermo-Sensitive Polyurethane Membrane with Controllable Water Vapor Permeation for Food Packaging
}

\author{
Hu Zhou', Huanhuan Shi ${ }^{1}$, Haojun Fan ${ }^{1,2, *}$, Jian Zhou ${ }^{1}$, and Jixin Yuan ${ }^{2}$ \\ ${ }^{1}$ College of Light Industry, Textile and Food Engineering, Sichuan University, Chengdu, 610065, P.R. China \\ ${ }^{2}$ College of Chemistry and Materials Science, Wenzhou University, Wenzhou, 325024, P.R. China
}

Received October 21, 2008; Revised November 9, 2008; Accepted November 27, 2008

\begin{abstract}
The size and shape of free volume (FV) holes available in membrane materials control the rate of gas diffusion and its permeability. Based on this principle, a segmented, thermo-sensitive polyurethane (TSPU) membrane with functional gate, i.e., the ability to sense and respond to external thermo-stimuli, was synthesized. This smart membrane exhibited close-open characteristics to the size of the FV hole and water vapor permeation and thus can be used as smart food packaging materials. Differential scanning calorimetry (DSC), dynamic mechanical analysis (DMA), positron annihilation lifetimes (PAL) and water vapor permeability (WVP) were used to evaluate how the morphological structure of TSPU and the temperature influence the FV holes size. In DSC and DMA studies, TSPU with a crystalline transition reversible phase showed an obvious phase-separated structure and a phase transition temperature at $53{ }^{\circ} \mathrm{C}$ (defined as the switch temperature and used as a functional gate). Moreover, the switch temperature $\left(T_{s}\right)$ and the thermal-sensitivity of TSPU remained available after two or three thermal cyclic processes. The PAL study indicated that the FV hole size of TSPU is closely related to the $T_{s}$. When the temperature varied cyclically from $T_{s}-10^{\circ} \mathrm{C}$ to $T_{s}+10^{\circ} \mathrm{C}$, the average radius $(R)$ of the FV holes of the TSPU membrane also shifted cyclically from 0.23 to $0.467 \mathrm{~nm}$, exhibiting an "open-close" feature. As a result, the WVP of the TSPU membrane also shifted cyclically from 4.30 to $8.58 \mathrm{~kg} / \mathrm{m}^{2} \cdot \mathrm{d}$, which produced an "increase-decrease" response to the thermo-stimuli. This phase transition accompanying significant changes in the FV hole size and WVP can be used to develop "smart materials" with functional gates and controllable water vapor permeation, which support the possible applications of TSPU for food packaging.
\end{abstract}

Keywords: thermo-sensitive polyurethane, smart materials, functional gate, water vapor permeability, food packaging.

\section{Introduction}

How to control the water concentration of packaged food is very important for requirements and designs in food packaging. In many cases, food must contain suitable water so as to remain its taste, colure, concentration and other qualities. For packaged food, it is easy to maintain the water content and keep it unchanged in the period of storage, but it is very difficult to change the water concentration, for example increase or decrease the water content in storage, ${ }^{1}$ because there is few packaging materials possessing this smart "breathability", i.e. with controllable water vapor permeation, except thermo-sensitive polyurethanes (TSPU) packaging materials.

As a matter of fact, polyethylene, poly(phenylene ether), nylon and polyurethane are all the most popular materials for packaging applications. The "breathability" of these membranes is mostly determined by the size and shape of

*Corresponding Author. E-mail: fanhaojun@163.com holes available in the polymer. ${ }^{2}$ But unfortunately, the size of holes in these non-porous membranes is too small to permit any gas/water vapor permeation, so once a completion of food packaging, the water concentration of food can never be adjusted, but it is not the case for TSPU. TSPU is defined as functional materials with the ability to sense and respond to external thermo-stimuli in a predetermined temperature range. Generally, these polymers consist of two phases, i.e. a thermally reversible phase and a fixed phase. Accordingly, TSPU has often been observed with block or segmented structure. In any case, the thermally reversible phase shows a phase transition temperature, which can be used as a switch temperature $\left(T_{s}\right) .^{3}$ It is well known that phase transition often accompanies great changes in physical properties of polymeric materials, ${ }^{4-6}$ especially a large increase of free-volume (FV) hole size and an enhanced micro-Brownian motion when heating through the phase transition temperature $\left(T_{s}\right)$, which provides a feasible approach to control the free-volume hole size. ${ }^{7,8}$

The working principle of TSPU for controllable water 
vapor permeation relies on its controllable FV holes size. When the environment temperature is lower than the $T_{s}$, the polymer chain is frozen and in glassy state, the microBrownian motion of macromolecules is limited and thus the FV hole size is too small to permit the water vapor penetration; When the environment temperature is higher than the $T_{s}$, the frozen polymer chains are released, and the increase of FV hole size together with the enhancement of microBrownian motion will cause the water vapor permeability of membranes to increase dramatically., ${ }^{9,10}$ When the temperature decreases to below $T_{s}$ again, the polymer chains are frozen again and thus the FV hole size also decreases. In this case, The $T_{s}$ seems to acts as a functional gate to control the FV hole size, and finally to control the water vapor permeation. The aim of this study is to develop "smart materials" with functional gates, furthermore, to provide an intelligent packaging material for food industry.

\section{Experimental}

Materials. Polycaprolactone diols (PCL, Daicel Co.) with molecular weight $10,000 \mathrm{~g} / \mathrm{mol}$ was dried at $100-120^{\circ} \mathrm{C}$ under 1-2 mmHg for $5 \mathrm{~h}$ before use. Extra pure grade of methylene diphenylene diisocyanate (MDI, Aldrich Chemical), 1,4-butanediol (BDO, Aldrich Chemical) were used to prepare TSPU.

Preparation of Thermo-Sensitive Polyurethane. A 500 $\mathrm{mL}$ round-bottom, four-necked separable flask equipped with a mechanical stirrer, nitrogen inlet, thermometer and condenser with a drying tube was used as a reactor to prepare the polyurethanes. Dried PCL and MDI, which would provide the switch temperature or the so-called functional gate in the polymer, and twofold mole ratio of MDI, were charged into the dried flask. The soft-segment-forming reaction was carried out at $80{ }^{\circ} \mathrm{C}$ for about $2.0 \mathrm{~h}$, followed by hard-segment-forming reaction with $\mathrm{MDI}$, BDO (mole ratio $1: 2$ ) at $75^{\circ} \mathrm{C}$ for $3.0 \mathrm{~h}$. The progress of reactions in preparing the NCO-terminated pre-polymers and chain extension step were monitored by measuring the isocyanate value. A standard di-n-butylamine back titration method was employed. $^{11}$

Instrumental Analysis. The phase transition temperature of the TSPU was measured by using a Dupont 9900 thermal analyzer with a DSC module, which was purged with nitrogen gas and quenched with liquid nitrogen. The TSPU samples $(5-10 \mathrm{mg})$ were heated in sealed aluminum pans and scanned from -50 to $200{ }^{\circ} \mathrm{C}$ then cooled from 200 to $-50{ }^{\circ} \mathrm{C}$ with a heating/cooling rate of $10{ }^{\circ} \mathrm{C} / \mathrm{min}$. The heating process runs two times to examine the effect of different thermal cyclic process on the segmental structure and properties. Dynamic mechanical properties were measured in the tensile mode at a fixed frequency of $2 \mathrm{~Hz}$ and under nitrogen gas purging by a DuPont 983 DMA, every testing repeats three times. To negate the DMA's dependence on the Pois- son ratio, a length-to-thickness ratio that was larger than 10 was used. The measured rectangular specimens were heated from -50 to $200{ }^{\circ} \mathrm{C}$ then cooled from 200 to $-50{ }^{\circ} \mathrm{C}$ using a heating or cooling rate of $5{ }^{\circ} \mathrm{C} / \mathrm{min}$. The data of the storage modulus, loss modulus and $\tan \delta$ were recorded.

The Positron Annihilation Lifetime (PAL) of TSPU was determined by detecting the prompt r-ray $(1.28 \mathrm{MeV})$ from the nuclear decay that accompanied the emission of a positron from the $\mathrm{Na}-22$ radioisotope and the annihilation $\mathrm{r}$ ray $(0.511 \mathrm{MeV})$. A fast-fast coincidence circuit of the PAL spectrometer with a lifetime resolution of 290 ps as monitored with a Co-60 source was used to record all PAL spectra. The spectrum $(1,000,000$ counts) was collected at each temperature for a complete data analysis of lifetime distribution. The count rate was 200 counts/s and the channel width was 61 ps. Both RESOLUTION and POSITRONFIT programs in the PATFIT88 package were employed for data analysis. $^{12}$

TSPU Membrane Preparation. Membranes were prepared by casting TSPU solution on a polytetrafluoroethylene (PTEF) plate with $5 \mathrm{~cm} \times 5 \mathrm{~cm} \times 0.2 \mathrm{~mm}$. After standing at $60{ }^{\circ} \mathrm{C}$ for $10 \mathrm{~h}$, the membranes were further dried at $100{ }^{\circ} \mathrm{C}$ under vacuum condition for the next $10 \mathrm{~h}$.

Measurement of WVP. WVP of the TSPU membrane was measured according to ASTM method E 96 Desiccant method, i.e. an open cup containing desiccant was sealed with the specimen membrane in such a manner that the cup mouth defined the area of the specimen exposed to the vapor pressure of the environment. The edges of the specimen were thoroughly sealed to prevent the passage of water vapor into, out of, or around the specimen edges or any portion thereof. Then the assembly was placed in a test chamber at the different temperatures of $15,25,35,45,55,65$, and $75{ }^{\circ} \mathrm{C}$ with a constant relative humidity of $50 \%$. In addition, in order to investigate the WVP reversibility of TSPU membrane, the WVPs of TSPU membranes were tested again from 75 to $15^{\circ} \mathrm{C}$. Finally, the weight change of permeation cup with the specimen was recorded after $24 \mathrm{~h}$ and the WVP was calculated by the following equation. The degree of water vapor permeability was expressed as $\mathrm{kg} /$ $\mathrm{m}^{2} \cdot \mathrm{d}$.

$$
\mathrm{WVP}=\frac{a_{1}-a_{2}}{S}
$$

Where, $\left(a_{1}-a_{2}\right)$ : weight change of permeation cup with the specimen $(\mathrm{kg}), S$ : area of permeation $\left(\mathrm{m}^{2}\right)$.

\section{Results and Discussion}

The Segmental Structure and Phase Transition Temperature of TSPU. The segmented structure and phase transition temperature of polymers can be characterized by DSC and DMA analysis. ${ }^{13,14}$ In order to examine the effect of different thermal cyclic process on the segmental structure and 
the thermal sensitive properties, every heating process runs two or three times. Figure 1 is the DSC patterns of TSPU.

On the first-heating curve, a peak at $53{ }^{\circ} \mathrm{C}$ and a point of inflection at $179^{\circ} \mathrm{C}$, corresponding to the crystalline melting transition of soft and hard segment respectively, were found implying that TSPU prepared in this study is phaseseparated into a soft segment and a partially hard segment, and the incompatible soft and hard segments show obviously independent phases and different phase transition temperatures. On the second-heating curve, similar phase transitions and phase transition temperature are observed, which means that different thermal cyclic process (involving a heating process and a cooling process) has little effect on the segmented structure and the thermal sensitive properties of TSPU. Above conclusions can be further determined by DMA analysis.

Just as mentioned above, phase transition often accompanies a significant change of dynamic mechanical properties, on DMA patterns of TSPU, there are two significant changes of $E^{\prime}, E^{\prime \prime}$ and $\tan \delta$ for any case, one appears at about $53{ }^{\circ} \mathrm{C}$,

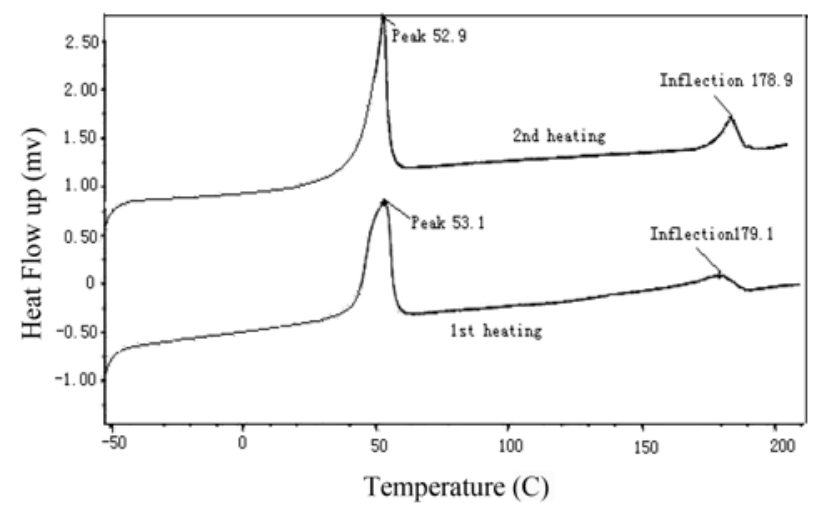

Figure 1. DSC curves of TSPU with a crystalline soft segment.

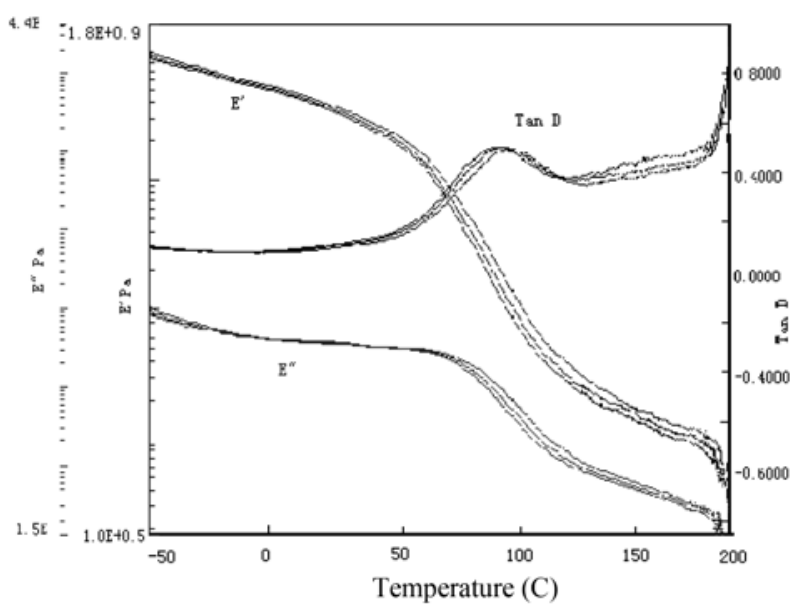

Figure 2. Storage modulus $\left(E^{\prime}\right)$, loss modulus $\left(E^{\prime \prime}\right)$ and tan $\delta$ of TSPU as a function of temperature and different thermal cyclic process. and another at $180{ }^{\circ} \mathrm{C}$ which correspond to the phase transition of the soft segment and the hard segment (Figure 2). DMA analysis also reveals that TSPU shows the segmental phase-separated structure and still remains its phase-separated structure and dynamical mechanical properties in every thermal cyclic process.

The Free Volume and the Size Scale of Micro-pores in TSPU Membranes. The Positron Annihilation Lifetime Spectroscopy has been widely employed to study the microstructure of macromolecular, especially to determine the free volume hole size of nano-scale (0.1-1.5 nm) in polymers. ${ }^{12}$ From the analysis of Positron Annihilation Lifetime Spectroscopy, we can obtain the data of ortho-positronium (o-Ps) lifetime $\left(\tau_{3}\right)$ and $o$-Ps intensity $\left(I_{3}\right)$, and then the average radius $(R)$ of free volume holes can be calculated by the following semi-empirical equation. ${ }^{15}$

$$
\tau_{3}=\frac{1}{2}\left[1-\frac{R}{R_{0}}+\frac{1}{2 \pi} \sin \left(\frac{2 \pi R}{R_{0}}\right)\right]^{-1}
$$

Where, $R_{0}=R+\delta_{R} ; \delta_{R}=0.166 \mathrm{~nm}$, which is the fitted empirical electron layer thickness. ${ }^{16}$

Furthermore, the average size of free volume holes $\left(V_{h}\right)$ and the fractional free volume $f_{v}(\%)$ can be calculated by eqs. (3) and (4) respectively.

$$
\begin{aligned}
& V_{h}=\frac{4}{3} \pi R^{3} \\
& f_{v}=C V_{h} I_{3}
\end{aligned}
$$

Where, $C$ is empirically determined to be 0.0018 from the specific volume data. Figure 3 , describes the $\tau_{3}, I_{3}, R$ and $f_{v}$ of TSPU as a function of temperature.

The free volume hole size and the fractional free volume of polymer can be characterized by the ortho-positronium (o-Ps) lifetime $\left(\tau_{3}\right)$ and the o-Ps intensity $\left(I_{3}\right)$. On one hand, the $o$-Ps lifetime $\left(\tau_{3}\right)$ is related to the size scale of micropores, and higher $\tau_{3}$ value means larger average radius $(R)$ of free volume holes. Correspondingly, the average size of free volume holes $\left(V_{h}\right)$ also shows a larger value. On the other hand, the $o$-Ps intensity $\left(I_{3}\right)$ is proportional to the amount of free volume holes, and higher $I_{3}$ value means larger amount of free volume holes. ${ }^{12,15}$ In other words, the fractional free volume $\left(f_{v}\right)$ will take on a higher value. As shown in Figure 3, when the temperature is lower than the switch temperature $\left(T_{s}\right)$, sample shows a relative lower $\tau_{3}, R$, $I_{3}$ or $f_{v}$ value. This is probably because that the TSPU is in a well-organized glassy state and all movements of macromolecule chains are almost frozen at this moment. When the temperature exceeds the $T_{s}$, TSPU undergo a change from the glassy state to the rubbery state. Just acting as a functional gate, phase transition temperature leads to dramatic changes in $\tau_{3}, R, I_{3}$ or $f_{v}$. Typically, when the temperature varies $\pm 10{ }^{\circ} \mathrm{C}$ around $T_{s}$, the average radius has shifted 

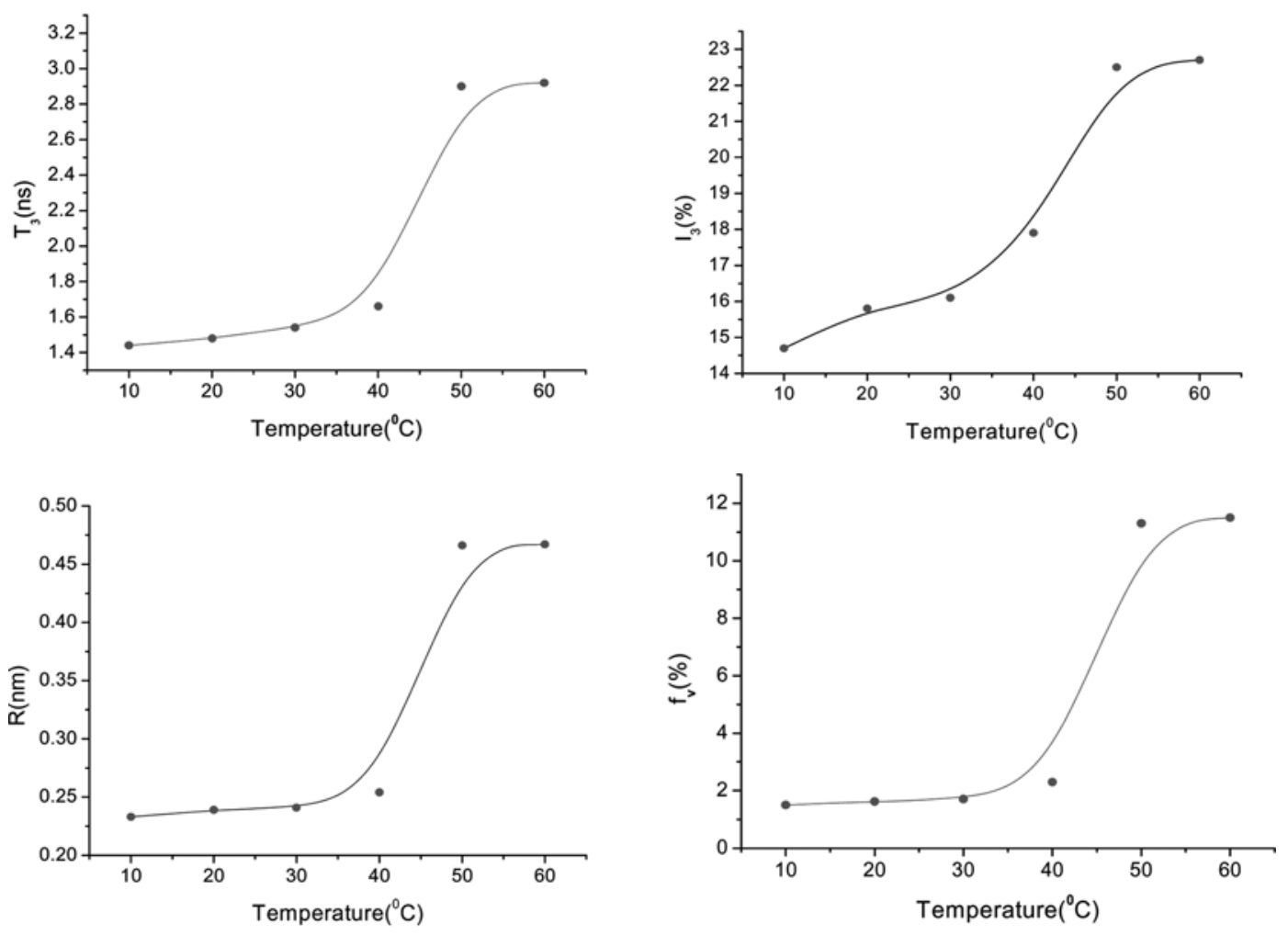

Figure 3. $\tau_{3}, I_{3}, R$ and $f_{v}$ of TSPU as a function of temperature.

from 0.23 to $0.467 \mathrm{~nm}$ and become larger than that of the water vapor $\left(R_{w}=0.4 \mathrm{~nm}\right) .{ }^{16}$ Lower $\tau_{3}, R, I_{3}$ or $f_{v}$ at lower temperature and higher $\tau_{3}, R, I_{3}$ or $f_{v}$ at higher temperature imply that TSPU membranes have good moisture barrier effect at lower temperature but a higher WVP at higher temperature.

The Water Vapor Permeability of TSPU Membranes. Figure 4, shows the water vapor permeability of TSPU membranes as a function of temperature. Although the WVP of sample increases with an increase of temperature, the increasing tendency is quite different in different temperature range.

As shown in Figure 4, when the temperature is lower than $45^{\circ} \mathrm{C}$, TSPU is in the glassy state and the FV hole size is lower than $0.23 \mathrm{~nm}$. Therefore, the WVP of sample shows a very low value and little change; When the temperature rises from 45 to $55^{\circ} \mathrm{C}$, TSPU has undergone a phase transition from the glassy state to the rubbery state during this process, the increased free volume hole size $(0.467 \mathrm{~nm})$ and the enhanced micro-Brownian motion in the polymer lead the WVP of TSPU increase from $4.30 \mathrm{~kg} / \mathrm{m}^{2} \cdot \mathrm{d}$ at $45^{\circ} \mathrm{C}$ to $8.58 \mathrm{~kg} / \mathrm{m}^{2} \cdot \mathrm{d}$ at $55^{\circ} \mathrm{C}$, showing a significant improvement as high as $199 \%$ (curve a). Another interesting result is that when the temperature backs to lower temperature (curve b), the WVP also backs to the low value, for example, when the temperature decreases from 55 to $45^{\circ} \mathrm{C}$, the WVP decrease from 8.65 to $4.38 \mathrm{~kg} / \mathrm{m}^{2} \cdot \mathrm{d}$, showing a good reversibility to

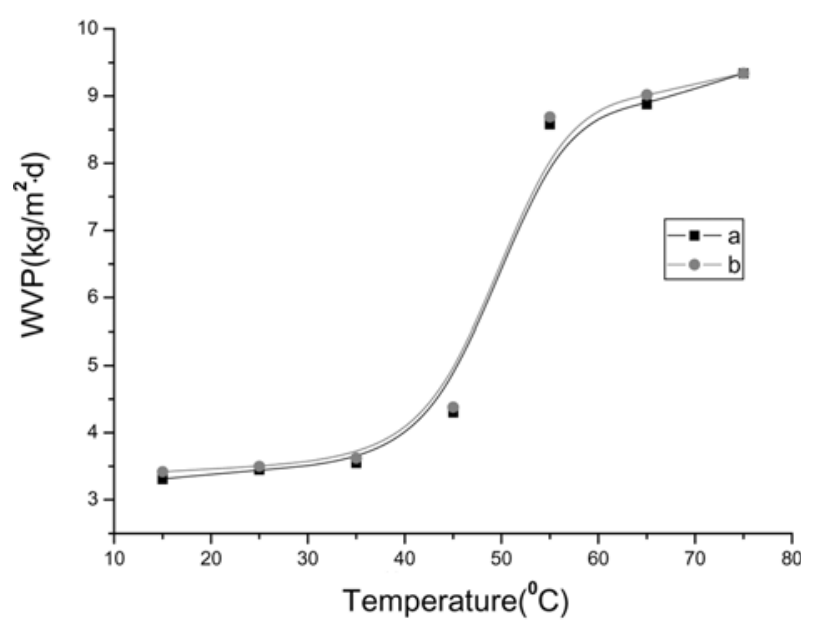

Figure 4. The WVPs of TSPU membranes as a function of temperature (a: the test temperature increases from 15 to $75^{\circ} \mathrm{C}$; b: the test temperature decreases from 75 to $15^{\circ} \mathrm{C}$ ).

thermal-stimuli. In this case, the $T_{s}$ acts as a functional gate and controls the WVP to increase or decrease.

Possible Applications of TSPU for Food Packaging Materials. It is believed that the size and shape of free volume holes available in polymeric membranes control the rate of gas diffusion and its permeability, ${ }^{9,17-19}$ so how to control the free volume hole size is the key index to achieve controllable water vapor permeation for packaging materi- 
als. TSPU membranes prepared in this study has the ability to sense and respond to external thermo-stimuli and give a changeable free volume hole size from 0.23 to $0.467 \mathrm{~nm}$. In practical applications, the water concentration of food packaged with TSPU membranes can be controlled by the external temperature and humidity. For example, when a enclosed food is placed in an environment with high humidity and high temperature $\left(>T_{s}\right)$, the outside water can be driven to enter the package because of the enlarged FV hole size and the presence of concentration pressure of water vapor, once the adsorption equilibrium establishes, the packaged food is taken away form the above environment, the polymer is again in glassy state and the "opened" FV holes in polymer seem to "close", therefore, the penetration process of water vapor ends automatically; whilst when the enclosed wet-food is placed in an environment with lower humidity and high temperature $\left(>T_{s}\right)$, in adverse, the intrinsic water of food will diffuse to the outside of packaging and thus the inner food is dried without re-opening the packaging. Working on this principle, the water concentration of food packaged with TSPU membranes will respond automatically to the stimuli of external temperature and humidity and show controllable features.

\section{Conclusions}

(1) TSPU prepared in this study shows phase-separated behavior and switch temperature $\left(T_{s}\right)$ in the designed temperature range. PAL study indicates that the average radius $(R)$ of free volume $(\mathrm{FV})$ holes in TSPU show dramatic changes when the temperature exceeds the $T_{s}$.

(2) The size of FV hole and WVP of TSPU can be controlled by the $T_{s}$; to be specific, when the temperature exceeds $T_{s}$, the size of FV hole increases from 0.23 to $0.467 \mathrm{~nm}$, as a result, the WVP of TSPU membrane increases from 4.30 to $8.58 \mathrm{~kg} / \mathrm{m}^{2} \cdot \mathrm{d}$; whilst when the temperature decreases to below $T_{s}$, the size of FV hole and WVP also decrease to below $0.23 \mathrm{~nm}$ and $4.38 \mathrm{~kg} / \mathrm{m}^{2} \cdot \mathrm{d}$, showing "openclose" and reversibility characteristics.

(3) The WVP of TSPU membrane responds to external thermo-stimuli automatically and shows controllable water vapor permeation, providing possible applications for food packaging.

Acknowledgments. The authors wish to acknowledge the financial support of the Program for New Century Excellent Talents in University (China).

\section{References}

(1) L. E. Gerlowski, Barrier Polymers and Structures, 423, 177 (1990).

(2) Y. C. Jean, J. P. Yuan, J. Liu, and H. J. Yang, J. Polym. Sci. Part B: Polym. Phys., 33, 2365 (1995).

(3) Y. Chen, Y. Liu, and H. J. Fan, et al., J. Membrane Sci., 287, 192 (2007).

(4) H. T. Lee and D. S. Lee, Macromol. Res., 10, 359 (2002).

(5) S. G. Kim and D. S. Lee, Macromol. Res., 10, 365 (2002).

(6) S. Y. Park and Y. H. Cho, Macromol. Res., 13, 156 (2005).

(7) H. M. Jeong, J. B. Lee, S. Y. Lee, and B. K. Kim, J. Mater. Sci., 35, 279 (2000).

(8) X. M. Ding, J. L. Hu, and X. M. Tao, Text. Res. J., 74, 39 (2004).

(9) S. Hayashi, N. Ishikawa, and C. Giordano, J. Coated Fabrics, 23, 74 (1993).

(10) H. J. Fan, L. Li, X. N. Fan, and B. Shi, J. Soc. Leath. Tech. Ch., 89, 121 (2005).

(11) D. J. David and H. B. Staley, Analytical Chemist of Polyurethanes, Wiley/Interscience, New York, 1969.

(12) B. Wang, M. Zhang, and J. M. Zhang, Phys. Lett. A, 262, 195 (1999).

(13) S. H. Kang, D. C. Ku, J. H. Lim, Y. K. Yang, N. S. Kwak, and T. S. Hwang, Macromol. Res., 13, 212 (2005).

(14) J. K. Yun, H. J. Yoo, and H. D. Kim, Macromol. Res., 15, 22 (2007).

(15) H. Nakanishi, S. J. Wang, and Y. C. Jean, Positron annihilation studies of fluids, S. C. Sharma, ed., World Science, Singapore, 1988.

(16) W. Y. Jeong and S. K. An, J. Mater. Sci., 36, 4797 (2001).

(17) H. M. Jeong, B. K. Ahn, S. M. Cho, and B. K. Kim, J. Polym. Sci. Part B: Polym. Phys., 38, 3009 (2000).

(18) B. K. Kim, S. Lee, and Y. M. Xu, Polymer, 37, 5781 (1993).

(19) B. K. Kim, S. Y. Lee, and J. S. Lee, Polymer, 39, 2803 (1998). 